Thrombosen an Arterien und Venen der Netzhaut

\title{
Erblindungsgefahr: Gefäßverschluss im Auge
}

\author{
Wie gravierend sich ein Verschluss von Netzhautarterien oder -venen auswirkt, hängt vor \\ allem davon ab, ob das zentrale Gefäß oder ein Seitenast betroffen ist. Chronische Folgen \\ kann insbesondere eine retinale Zentralvenenthrombose nach sich ziehen.
}

\begin{abstract}
Verschlüsse von Retinalarterien betreffen zu fast $60 \%$ die Zentralarterie, $\mathrm{zu}$ fast $40 \%$ Seitenäste und nur selten zilioretinale Gefäße. Während sich eine kurzfristige Perfusionsunterbrechung in einer Amaurosis fugax äußert, führt ein anhaltender Verschluss zu einem Retinainfarkt. Astverschlüsse haben weniger deletäre Folgen als Zentralarterienverschlüsse. Die Ischämietoleranz der Retina beträgt 105 Minuten, wie Prof. Sebastian Schellong, Dresden, ausführte.
\end{abstract}

\section{Arterienverschluss: Plötzlicher schmerzloser Visusverlust}

Charakteristisches Symptom eines zentralen Netzhautarterienverschlusses ist ein plötzlicher, anhaltender und schmerzloser Visusverlust auf einem Auge. Ein Astverschluss kann auch nur mit einem teilweisen Verlust des Sehvermögens einhergehen oder auch asymptomatisch ablaufen. Aufgrund der Anatomie der Netzhautgefäße verläuft die Linie zwischen gestörtem und intaktem Sehvermögen immer horizontal. Bei der Fundoskopie fällt die Makula dem Au-

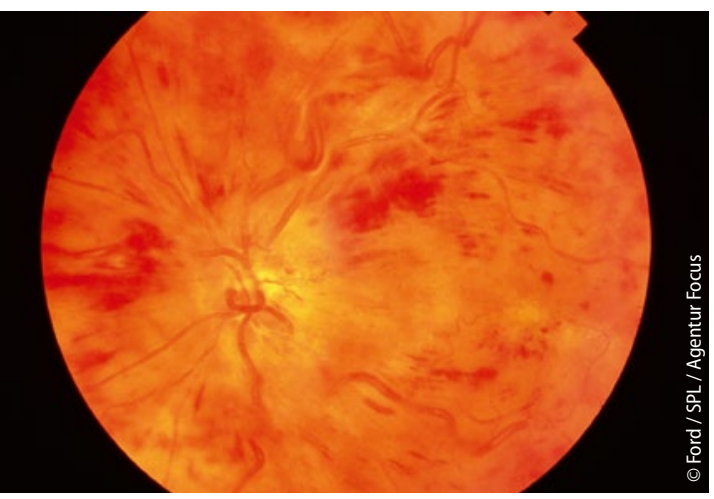

Fundoskopie bei Verschluss der Zentralvene: Papillenödem, Blutungen und gelbe Cotton-wool-Flecken. genarzt als kräftiger roter Fleck auf (red cherry sign). Es kann aber auch sein, dass bei einem arteriellen Verschluss gar keine Veränderungen am Augenhintergrund sichtbar sind.

Die meisten Retinaarterienverschlüsse sind Manifestationen einer kardiovaskulären Systemerkrankung. Pathophysiologisch handelt es sich am häufigsten um eine Embolie. Selten können eine autochthone Arteriosklerose, Vasospasmen oder eine Vaskulitis vorliegen.

Die Therapiemöglichkeiten sind unbefriedigend. Man kann durch Bulbusmassage eine reaktive Hyperämie erzeugen oder den intraokulären Druck durch Betablocker oder Acetazolamid senken. Eine kathetergeführte Thrombolyse hat sich nicht durchgesetzt, vor allem wegen der Gefahr von Retinablutungen. Die wichtigste Aufgabe für den Internisten ist die Umfelddiagnostik, d.h. die Erfassung anderer kardiovaskulärer Erkrankungen und Risikofaktoren sowie die Suche nach der Emboliequelle.

\section{Venenthrombosen: Langzeitproblem Neovaskularisationen}

Auch Venenthrombosen können die zentrale Netzhautvene oder Äste betreffen. Außerdem unterscheidet man eine ischämische Verlaufsform (25\%) und eine nicht-ischämische (75\%). Die Venenthrombose führt zu einer perakuten Drainagestörung, die ein Makulaödem und eine Okklusion der Venen hervorruft. Außerdem können sich ein Glaukom und eine Netzhautablösung entwickeln.

Langfristige Probleme aber schaffen die Neovaskularisation und Glaskörpereinblutungen aufgrund der Drainagestörung. Diese Sekundärprobleme machen die Retinavenenthrombose zu einem chronischen Krankheitsbild und zur zweithäufigsten Erblindungsursache nach der diabetischen Retinopathie.

Bei der ischämischen Zentralvenenthrombose tritt ein plötzlicher und anhaltender einseitiger Visusverlust auf, der auch etwas schmerzhaft ist. Die nichtischämische Zentralvenenthrombose äußert sich in einem intermittierenden mäßigen und schmerzlosen einseitigen Verlust des Sehvermögens. Ist nur ein Venenast thrombosiert, kommt es höchstens $\mathrm{zu}$ einem leichten umschriebenen Visusverlust, manchmal entwickeln sich auch gar keine Symptome.

Bei einer ischämischen Zentralvenenthrombose zeigt die Fundoskopie aufgrund der Drainagestörung schwere Netzhautblutungen in allen vier Quadranten, ein deutliches Makulaödem, ein Papillenödem und aufgetriebene Venen. Papillenschwellung und aufgetriebene Venen findet man auch beim nichtischämischen Zentralvenenverschluss. Die Netzhautblutungen sind hier jedoch variabel ausgeprägt. Lokale Netzhautblutungen kennzeichnen die Thrombose eines Retinavenenastes.

Auch die Retinavenenthrombose steht in Assoziation mit klassischen Risikofaktoren wie Hypertonie, Diabetes oder Hyperlipidämie. Ganz selten kann sie in Zusammenhang mit einem Antiphospholipidsyndrom oder mit einer Hyperhomozysteinämie auftreten.

Behandelt wird die Venenthrombose mit intraokulären Injektionen von Triamcinolon oder Dexamethason sowie mit VEGF-Antikörpern, um der Neovaskularisation vorzubeugen. Auch ein Hypertonus sollte gut eingestellt werden.

- Dr. med. Angelika Bischoff

- Symposium „Atypische Thrombosen", DGIM-Kongress Mannheim, 11. April 2016 